\title{
EXTENSIONS OF ABELIAN GROUPS OF FINITE RANK
}

\author{
S. A. KHABBAZ AND E. H. TOUBASSI
}

ABSTRACT. Every abelian group $X$ of finite rank arises as the middle group of an extension $e: 0 \rightarrow F \rightarrow X \rightarrow T \rightarrow 0$ where $F$ is free of finite rank $n$ and $T$ is torsion with the $p$-ranks of $T$ finite for all primes $p$. Given such a $T$ and $F$ we study the equivalence classes of such extensions which result from stipulating that two extensions $e_{i}: 0 \rightarrow F \rightarrow X_{i} \rightarrow T \rightarrow 0, i=1,2$, are equivalent if $e_{1}=\beta e_{2} \alpha$ for $\alpha \in A u t(T)$ and $\beta \in$ Aut $(F)$. We reduce the problem to $T p$-primary of finite rank, where in the one case $T$ is injective, and in the other case $T$ is reduced. Suppose $T=\Pi_{i=1}^{m} T_{i}$. In our main theorems we prove that in each case these equivalence classes of extensions are in 1-1 correspondence with the equivalence classes of $n$-generated subgroups of $E$ where $E=\prod_{i=1}^{m} E_{i}, E_{i}=\operatorname{End}\left(T_{i}\right)$. Two $n$-generated subgroups of $E$ will be called equivalent if one can be mapped onto the other by an automorphism of $E$.

1. With few exceptions our notation will be that of [1].

One of the difficulties in studying groups of extensions $\operatorname{Ext}(A, B)$ is that the same group may appear as a middle group in two distinct elements of $\operatorname{Ext}(A, B)$. With this in mind we consider the action of the rings $\operatorname{End}(A)$ and $\operatorname{End}(B)$ on $\operatorname{Ext}(A, B)$. In the problem at hand we are interested in the equivalence classes of $\operatorname{Ext}(T, F)$ induced by the action of automorphisms of $T$ and $F$ where $T$ is a torsion group with its $p$-ranks finite for all primes $p$ and $F$ a free abelian group of rank $n$. Since $\operatorname{Ext}(T, F)=\Pi_{p} \operatorname{Ext}\left(T_{p}, F\right)$, where $T_{p}$ is the $p$-primary component of $T$, one may restrict himself to the case when $T$ is $p$-primary. (See the remark following Theorem 1.2.) Since $T$ is the direct sum of an injective group of finite rank and one which is finite we may treat each case separately. We now state our main theorems.

Theorem 1.1. Let $T$ be an injective p-primary group of rank $m$. Then the equivalence classes of extensions of the form $0 \rightarrow F \rightarrow X \rightarrow T \rightarrow 0$ are in 1-1 correspondence with the equivalence classes of n-generated subgroups of $E$.

Presented to the Society, January 17, 1974; received by the editors October 5 , 1973 and, in revised form, May 3, 1974.

AMS (MOS) subject classifications (1970). Primary 20K35, $20 \mathrm{~J} 05$.

Key words and phrases. Module of extensions, finite rank, homological methods in group theory. 
In the other case we have

Theorem 1.2. Let $T$ be a reduced p-primary group of finite rank. Then the equivalence classes of extensions of the form $0 \rightarrow F \rightarrow X \rightarrow T \rightarrow 0$ are in 1-1 correspondence with the equivalence classes of n-generated subgroups of $T$.

Remark. In the above theorems when $T$ is not primary one must consider a sequence of such classes, one for each prime. Alternatively, Theorems 1.1 and 1.2 hold if one replaces "p-primary" in them by "torsion" and interprets rank in the sense of [2], [3]. When $T$ is reduced and torsion an $n$-generated subgroup of $T$ is just a subgroup of $T$ of rank at most $n$ (over $Z$ ) in the sense of [2], [3].

We begin with a proposition of interest, the proof of which is easy and will be omitted.

Proposition 1.3. (a) Let $M$ be a torsion group of rank one. Then $\operatorname{Ext}(M, Z)$ is a free $\operatorname{End}(M)$-module of rank one.

(b) Let $t$ be an integer not exceeding s. Then $\operatorname{Ext}\left(Z\left(p^{t}\right), Z\left(p^{s}\right)\right)$ is a free $\operatorname{End}\left(Z\left(p^{t}\right)\right)$-module of rank one.

Throughout the remainder of the paper we shall use the following notation. Let $T=\Pi_{i=1}^{m} T_{i}, E_{i}=\operatorname{End}\left(T_{i}\right), E=\Pi_{i=1}^{m} E_{i}, V=Q \otimes F, S=\operatorname{End}(T)$ and $R=$ End $(F)$. We now give an outline of

Proof ${ }^{1}$ of Theorem 1.1. The 1-1 correspondence of Theorem 1.1 is a composite of three 1-1 correspondences. The first of these makes correspond to the equivalence class of $e \in \operatorname{Ext}(T, F)$, an equivalence class of $n \times m$ matrices of $p$-adic integers in $\operatorname{Hom}(T, V / F)$. Here two $n \times m$ matrices are equivalent if one is obtained from the other by multiplying on the left by an $n \times n$ invertible matrix of integers and on the right by an $m \times m$ invertible matrix of $p$-adic integers. By taking transposes we can view the matrix in $\operatorname{Hom}(F, E)$. Our final correspondence sends the equivalence class of matrices in $\operatorname{Hom}(F, E)$ to the class of $n$-generated subgroups of $E$ as in Theorem 1.1 by taking the class of the image of $F$ in $E$.

We now prove these 1-1 correspondences. Our next proposition proves the first correspondence and in fact is a generalization of that result.

Proposition 1.4. $\operatorname{Ext}(T, F)$ and $\operatorname{Hom}(T, V / F)$ are isomorphic $R-S-b i$ modules.

${ }^{1}$ The authors are grateful to the referee, whose careful reading of the paper resulted in an improvement in the proof of Theorem 1.1. 
Proof. Apply the functor $\operatorname{Hom}(T,-)$ to the exact sequence $0 \rightarrow F \rightarrow$ $V \rightarrow V / F \rightarrow 0$. This results in a group isomorphism $\theta: \operatorname{Hom}(T, V / F) \cong$ $\operatorname{Ext}(T, F)$, the map $\theta$ being the connecting homomorphism. Since $\theta$ is natural in $T$, i.e. $\theta(\sigma \delta)=\theta(\sigma) \delta$ for $\delta \in S$ and $\sigma \in \operatorname{Hom}(T, V / F)$, it is a map of right $S$-modules. It remains to show that it is also a map of left $R$-modules.

Since every endomorphism of a free abelian group of finite rank is a sum of automorphisms it suffices to show that $\theta(\rho \phi)=\rho \theta(\phi)$ holds for the units $\rho$ in $R$ and elements $\phi$ in $\operatorname{Hom}(T, V / F)$. Now $\theta(\phi)$ is represented by the sequence

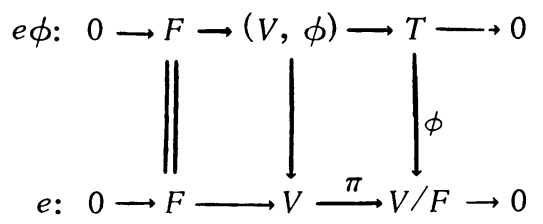

where $(V, \phi)=\{(x, t) \mid \pi(x)=\phi(t)\}$. Similarly $\theta(\rho \phi)$ can be represented by a sequence

$$
0 \rightarrow F \rightarrow(V, \rho \phi) \rightarrow T \rightarrow 0
$$

where $(V, \rho \phi)=\{(x, t) \mid \pi(x)=\rho \phi(t)\}$. We remark here that we are abusing the notation by referring to several homomorphisms as $\rho$. Note that $\rho: F \rightarrow F$ extends uniquely to an automorphism from $V \rightarrow V$ which in turn induces a homomorphism from $V / F \rightarrow V / F$. We shall refer to these as well as the inclusion $F \rightarrow(V, \rho \phi)$ given by $y \rightarrow(\rho(y), 0)$ as $\rho$. Since the diagram

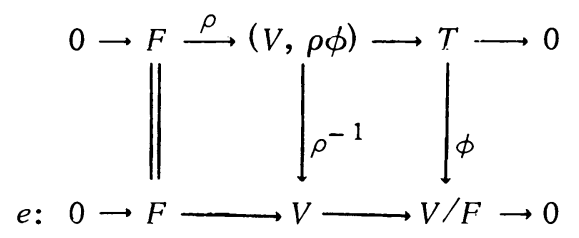

commutes, where $\rho^{-1}(x, t)=\rho^{-1}(x)$, we conclude that $e \phi=\theta(\phi)$ can be represented by (1). Using this and the sequence (1) we have the commutative diagram:

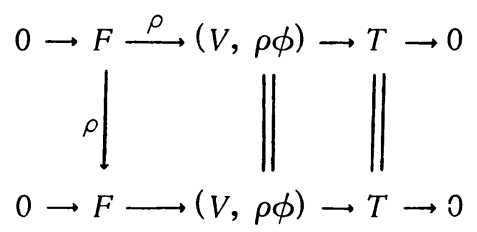

Since the top row here is $\theta(\phi)$, the bottom row must be $\rho \theta(\phi)$. By definition 
however, the bottom row is $\theta(\rho \phi)$. Thus $\theta(\rho \phi)=\rho \theta(\phi)$ as required.

Remark. We note that the above proof uses only the facts that $T$ is torsion and that every endomorphism of a free abelian group of finite rank is a sum of automorphisms.

Before we get into our second correspondence we recall the following facts. If $A=A_{1} \oplus \cdots \oplus A_{r}$ and $B=B_{1} \oplus \cdots \oplus B_{s}$ with injections $\alpha_{j}$ : $A_{j} \rightarrow A$ and projections $\beta_{i}: B \rightarrow B_{i}$, the map $\phi \rightarrow \beta_{i} \phi \alpha_{j}$ is an isomorphism of $\operatorname{Hom}(A, B)$ onto the group of matrices $M_{s \times r}(A, B)$ having as entries in the $(i, j)$ position the elements of $\operatorname{Hom}\left(A_{j}, B_{i}\right)$. In particular if $\Lambda=$ End $(A)$ then $\Lambda$ is isomorphic as a ring to $M_{r \times r}(A)$ and $\Sigma=\operatorname{End}(B)$ is isomorphic to $M_{s \times s}(B)$. Moreover the $\Sigma-\Lambda$-bimodule $\operatorname{Hom}(A, B)$ is isomorphic in the obvious sense to the $M_{s \times s}-M_{r \times r}$-bimodule $M_{s \times r}$. We now prove a generalization of our second correspondence.

Proposition 1.5. The $R-S$-bimodule $\operatorname{Hom}(T, V / F)$ is isomorphic to the $S$ - R-bimodule $\operatorname{Hom}(F, E)$.

Proof. Interpreting the above paragraph in the case when $T$ is an injective $p$-primary group of rank $m, E$ becomes a free module of rank $m$ over the $p$-adic integers $J$, and we obtain an isomorphism of the $R$ - $S$-bimodule $\operatorname{Hom}(T, V / F)$ with the $Z_{n \times n}-J_{m \times m}$-bimodule $J_{n \times m}$. Here we note that we are considering $V / F$ as a module over the endomorphism ring of $F$, not of $V / F$, but the correspondence described above remains valid. On the other hand, the $S-R$-bimodule $\operatorname{Hom}(F, E)$ is isomorphic to the $J_{m \times m}-Z_{n \times n}$-bimodule $J_{m \times n}$. Taking transposes one gets an isomorphis $m$, in the obvious sense, of the $R-S$-bimodule $\operatorname{Hom}(T, V / F)$ with the $S-R$-bimodule $\operatorname{Hom}(F, E)$. This concludes the proof.

Now we come to our final correspondence. This arises from the observation that an $n$-generated subgroup of $E$ is just a homomorphic image of $F$ in E. We state this as

Proposition 1.6. The elements $\phi, \phi^{\prime}$ in $\operatorname{Hom}(F, E)$ have equivalent images if and only if $\phi^{\prime}=\alpha \phi \beta$ for some $\alpha \in \operatorname{Aut}(E)$ and $\beta \in \operatorname{Aut}(F)$.

Proof. Note that if $\phi^{\prime}=\alpha \phi \beta$ then the images of $\phi$ and $\phi^{\prime}$ are equivalent since they are mapped onto each other by $\alpha$. Moreover, this correspondence is clearly onto. In order to prove that it is 1-1 we have to show that for $\phi, \phi^{\prime} \in \operatorname{Hom}(F, E)$ if $\phi(F) \cong \phi^{\prime}(F)$ under an automorphism $\sigma$ of $E$ then there exist automorphisms $\beta$ of $F$ and $\alpha$ of $E$ such that $\alpha \phi \beta=\phi^{\prime}$. Since $\phi(F)$ and $\phi^{\prime}(F)$ are finitely generated torsion-free groups they are free. Thus 
one can decompose $F$ in two ways; $F \cong \phi(F) \oplus F_{1} \cong \phi^{\prime}(F) \oplus F_{2}$ where $F_{1} \cong$ kernel $\phi$ and $F_{2} \cong$ kernel $\phi^{\prime}$. Note that $F_{1} \cong F_{2}$ since $\phi(F) \cong$ $\phi^{\prime}(F)$. It is easy to see now that one can induce an isomorphim $\beta$ of $F$ such that the diagram below commutes:

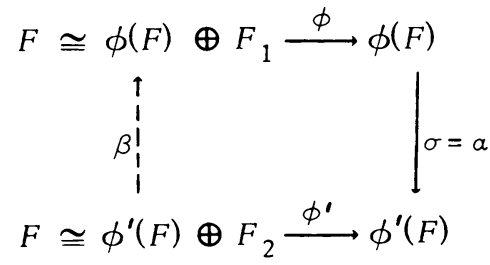

where $\alpha$ is set to be equal to $\sigma$. This completes the proof of Proposition 1.6 and Theorem 1.1 .

The above proof almost carries verbatim to prove Theorem 1.2. Unfortunately the $1-1$ relation of Proposition 1.6 breaks down. We circumvent this problem by replacing $F$ by $G=F / p^{s} F$ where $p^{s}$ is the minimal annihilator of $T$ and utilizing the module isomorphism of the following

Theorem 1.7. $\operatorname{Ext}(T, F)$ and $\operatorname{Ext}(T, G)$ are isomorphic as modules over the isomorphic rings $\operatorname{End}(F) \otimes \operatorname{End}(T)$ and $\operatorname{End}(G) \otimes \operatorname{End}(T)$.

Proof. First we show the isomorphism of the rings. Define $\phi$ : End $(F)$ $\otimes \operatorname{End}(T) \rightarrow \operatorname{End}(G) \otimes \operatorname{End}(T)$ by $\phi(\beta \otimes \alpha)=\bar{\beta} \otimes \alpha$ where $\bar{\beta}$ is the homomorphism making the diagram

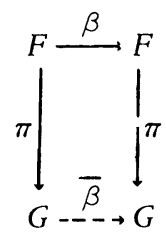

commute. It is straightforward to show that $\phi$ is an isomorphism. Since $p^{s} T=0$ observe that $F \stackrel{\pi}{\rightarrow} G$ induces an isomorphism of groups $\operatorname{Ext}(T, F)$ $\stackrel{\pi_{*}}{\longrightarrow} \operatorname{Ext}(T, G)$ where $e \rightarrow \pi e$. This isomorphism is a module isomorphism since $\pi_{*}(\beta e \alpha)=\pi \beta e \alpha=\bar{\beta} \pi e \alpha=\bar{\beta} \pi_{*}(e) \alpha$.

Proof of Theorem 1.2. By Theorem 1.7 it suffices to give the proof for $G$ in place of $F$. The proof of Theorem 1.1 applies here except for the obvious changes and the 1-1 relation of Proposition 1.6. To do that we suppose that $\phi(F)$ is generated by $w_{1}, \cdots, w_{k}$ independent elements. Let $a_{1}, \cdots, a_{k} \in G$

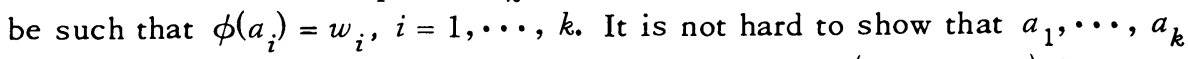
are independent elements each of $p$-height zero. Thus $\left\langle a_{1}, \cdots, a_{k}\right\rangle$ is a summand of $G$. Similarly the same holds for the preimage of the generators of $\phi^{\prime}(F)$. The proof is now completed as in Theorem 1.1. 


\section{BIBLIOGRAPHY}

1. L. Fuchs, Infinite abelian groups. Vols. I, II, Pure and Appl. Math., vol. 36, Academic Press, New York, 1970, 1973. MR 41 \#333.

2. S. A. Khabbaz and E. H. Toubassi, The module structure of $\operatorname{Ext}(F, T)$ over the endomorphism ring of T, Pacific J. Math. 54 (1975), 169-176.

3. -, $\operatorname{Ext}(A, T)$ as a module over $\operatorname{End}(T)$, Proc. Amer. Math. Soc. 48 (1975), 269-275.

DEPARTMENT OF MATHEMATICS, LEHIGH UNIVERSITY, BETHLEHEM, PENNSYLVANIA 18015

DEPARTMENT OF MATHEMATICS, UNIVERSITY OF ARIZONA, TUCSON, ARIZONA 85721 